\title{
Changes in Trends of Spondylitis in Korea Based on a Nationwide Database
}

\author{
Sung Hyun $\mathrm{Noh}^{1,2}$, Ho Yeol Zhang ${ }^{1,2}$, Sang Hoon Lee ${ }^{1,2}$, Jung Kyu Choi ${ }^{3}$, and Dong Kyu Chin ${ }^{4}$ \\ ${ }^{1}$ Department of Neurosurgery, National Health Insurance Service Ilsan Hospital, Goyang; \\ ${ }^{2}$ Department of Neurosurgery, Yonsei University College of Medicine, Seoul; \\ ${ }^{3}$ Department of Policy Research Affairs, National Health Insurance Service Ilsan Hospital, Goyang; \\ ${ }^{4}$ Department of Neurosurgery, Spine and Spinal Cord Institute, Gangnam Severance Hospital, Yonsei University College of Medicine, Seoul, Korea.
}

Infectious spondylitis is increasing with the growing population of older adults and immunocompromised individuals, the increased use of pain intervention, and magnetic resonance imaging (MRI). ${ }^{1}$ Cases of infectious spondylitis may be classified into the following three types: pyogenic, tuberculous, or nontuberculous (parasitic) spondylitis. ${ }^{2}$

Until the early 2000s, nontuberculous spondylitis and pyogenic spondylitis were rare: most cases of infectious spondylitis in Korea were thought to be tuberculous spondylitis because of the high prevalence of tuberculosis in the past. ${ }^{3}$ However, the prevalence of tuberculosis has been decreasing, which might lead to a consequent decrease in the incidence of tuberculous spondylitis. Meanwhile, however, pyogenic spondylitis has been increasing since the mid-2000s. The most common cause of pyogenic spondylitis is spontaneous, although iatrogenic causes are increasing. Accordingly, spinal surgeons have turned their attention to the diagnosis and treatment of pyogenic spondylitis.

Management of the growing number of cases of pyogenic spondylitis is complicated by the ambiguity of its symptoms and difficulty in differentiating between pyogenic and tuberculous spondylodiscitis. In pyogenic spondylitis especially, it is important to distinguish spontaneous pyogenic spondylitis caused by immune system compromise, such as that associated with dia-

\footnotetext{
Received: November 9, 2018 Revised: March 7, 2019

Accepted: March 10, 2019

Co-corresponding authors: Dong Kyu Chin, MD, PhD, Department of Neurosurgery, Spine and Spinal Cord Institute, Gangnam Severance Hospital, Yonsei University College of Medicine, 211 Eonju-ro, Gangnam-gu, Seoul 06273, Korea. Tel: 82-2-2019-3390, Fax: 82-2-3461-9229, E-mail: DKCHIN@yuhs.ac and

Ho Yeol Zhang, MD, PhD, Department of Neurosurgery, National Health Insurance Service Ilsan Hospital, 100 Ilsan-ro, Ilsandong-gu, Goyang 10444, Korea.

Tel: 82-31-900-0256, Fax: 82-31-900-0343, E-mail: hyzhang@nhimc.or.kr

-The authors have no potential conflicts of interest to disclose.

(C) Copyright: Yonsei University College of Medicine 2019

This is an Open Access article distributed under the terms of the Creative Commons Attribution Non-Commercial License (https://creativecommons.org/licenses/ by-nc/4.0) which permits unrestricted non-commercial use, distribution, and reproduction in any medium, provided the original work is properly cited.
}

betes, rheumatoid arthritis, or chronic steroid use, and pyogenic spondylitis due to iatrogenic factors, ${ }^{1}$ because their approaches to treatment and prognosis can differ. In addition, treatment of this disease is further impeded by delayed treatment onset, bacterial antibiotic resistance, and disease recurrence. Therefore, understanding trends in infectious spondylitis and its subtypes is important for the clinical decision making required to manage this disease.

We used customized data provided by the National Health Insurance Service to calculate the total prevalence and incidence of infectious spondylitis over a period of 11 years (2005 to 2015, IRB No. 2017-52). The National Health Information Database is a public database containing health care applications, health evaluations, sociodemographic variables, and mortality data for the entire Korean population that is maintained by the National Health Insurance Service. ${ }^{4}$ The data include information from more than 50 million people, and in 2014, the participation rate in national health screening programs was $74.8 \%{ }^{4}$ Patients with a previous history of infective spondylitis were excluded, and the incidence was measured based on newly diagnosed patients who were treated at an inpatient or outpatient clinic with infectious spondylitis between 2005 and 2015. From 2005 to 2015 , the total number of infectious spondylitis increased 2.7 times (from 11051 patients to 29796 patients). Also, the incidence of infectious spondylitis increased 2.5 times (from 23.3 patients to 58.4 patients). Among them, the number of patients with pyogenic spondylitis increased 2.6 times in 11 years (10323 patients in 2005 to 27321 patients in 2015) (Fig. 1A). The incidence rate of change of pyogenic spondylitis was much higher than that of tuberculous spondylitis. Furthermore, in 2005, the proportion of patients with infectious spondylitis who were younger than 60 years of age was $40.2 \%$; this proportion increased to $51.4 \%$ in 2015. Previously, infectious spondylitis had been noted to occur in older people with impaired immunity; however, this disease is now seen in younger patients, owing to iatrogenic factors (Fig. 1B). 

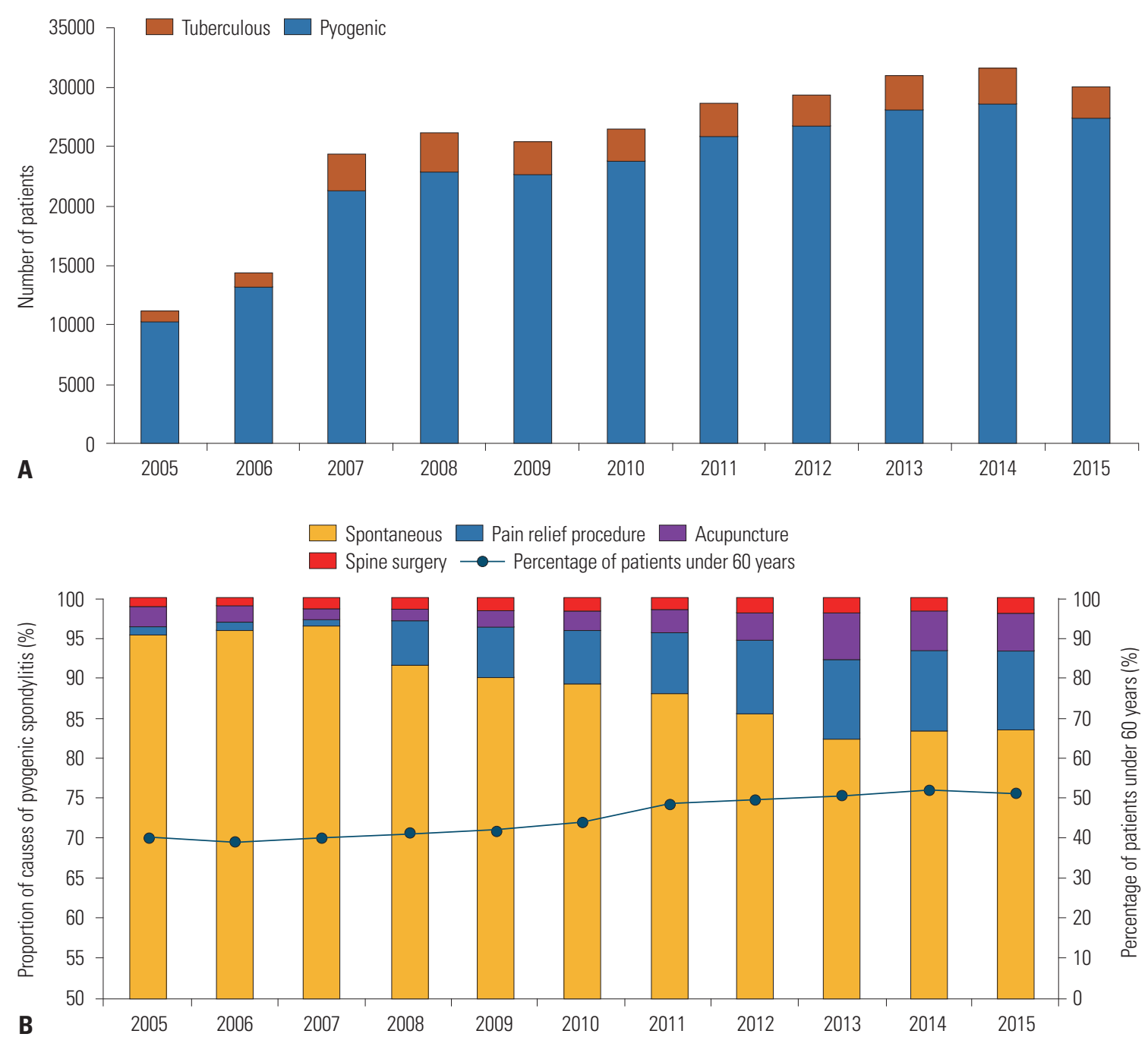

Fig. 1. Changes in trends of spondylitis in Korea. (A) Number of cases of infectious spondylitis in Korea from 2005-2015. Data were obtained from the National Health Insurance Service, Korea. (B) Proportions of the following causes of infectious spondylitis: spontaneous, pain relief procedures, acupuncture, and spinal surgery. Proportions of patients over 60 years of age with infectious spondylitis in Korea over 2005-2015.

The causes of pyogenic spondylitis are classified as iatrogenic factors or as spontaneous (no incidental reason and cause unknown). The iatrogenic factors are defined as various pain interventions and surgeries performed within 3 months of the diagnosis of pyogenic spondylitis, specifically acupuncture, pain relief procedures performed in the spinal and para-vertebral muscles, or spinal surgery. If two or more procedures have been performed, the one performed most recently was considered. In 2005, within 3 months of the diagnosis of pyogenic spondylitis, $1.0 \%$ patients had undergone pain relief procedures. In 2015, this proportion increased to $10.0 \%$, and epidural block was the most common pain relief procedure. Similarly, the proportion of patients who utilized acupuncture steadily increased from $2.6 \%$ in 2005 to $4.6 \%$ in 2015 (Fig. 1B). There are significant differences in pain relief procedures and acupuncture $(p<0.0001)$. Furthermore, in 2015, patients had most commonly undergone pain relief procedures (10\%), followed by acupuncture (4.6\%) and spinal surgery (1.7\%).
The number of cases of pyogenic spondylitis has been steadily increasing over the last decade; pyogenic spondylitis accounts for a significantly higher proportion of cases than tuberculous spondylitis does. In this study, we objectively confirmed the increasing prevalence of pyogenic spondylitis, which had otherwise been only anecdotally reported by spinal surgeons. While most cases of pyogenic spondylitis have an unknown cause, the rising incidence of pyogenic spondylitis has been closely mirrored by a steady increase in the number of pain interventions over the last 11 years. Although our research is limited to Korea, the change in the trends of spondylitis is not confined to Korea, and this issue is worth studying globally.

In pain relief procedures and acupuncture, careful consideration should be given to sterilization to prevent the occurrence of pyogenic spondylitis. In addition, if clinicians encounter a patient who has signs of infection after a pain procedure at an outpatient clinic, they should be able to determine the proper treatment through a quick diagnosis by considering the possi- 
bility of pyogenic spondylitis.

\section{AUTHOR CONTRIBUTIONS}

Conceptualization: Sung Hyun Noh, Dong Kyu Chin, Ho Yeol Zhang. Data curation: Jung Kyu Choi, Sang Hoon Lee. Formal analysis: Jung Kyu Choi, Sang Hoon Lee. Investigation: Dong Kyu Chin, Ho Yeol Zhang. Methodology: Sung Hyun Noh. Project administration: Sung Hyun Noh. Resources: Sung Hyun Noh. Software: Sung Hyun Noh. Supervision: Dong Kyu Chin, Ho Yeol Zhang. Validation: Dong Kyu Chin, Ho Yeol Zhang. Visualization: Dong Kyu Chin, Ho Yeol Zhang. Writing_original draft: Sung Hyun Noh. Writing—review \& editing: Sung Hyun Noh.

\section{ORCID iDs}

Sung Hyun Noh

https://orcid.org/0000-0003-2298-9035

Ho Yeol Zhang
Sang Hoon Lee Jung Kyu Choi

Dong Kyu Chin

https://orcid.org/0000-0003-4819-7917

https://orcid.org/0000-0001-5176-0534

https://orcid.org/0000-0002-9835-9294

\section{REFERENCES}

1. Vergne P, Trèves R. [Infectious spondylodiscitis. etiology, diagnosis, progression and treatment.] Rev Prat 1998;48:2065-71.

2. Kaufman DM, Kaplan JG, Litman N. Infectious agents in spinal epidural abscesses. Neurology 1980;30:844-50.

3. Lee Y, Kim BJ, Kim SH, Lee SH, Kim WH, Jin SW. Comparative analysis of spontaneous infectious spondylitis: pyogenic versus tuberculous. J Korean Neurosurg Soc 2018;61:81-8.

4. Seong SC, Kim YY, Khang YH, Park JH, Kang HJ, Lee H, et al. Data resource profile: the National Health Information Database of the National Health Insurance Service in South Korea. Int J Epidemiol 2017;46:799-800. 